\title{
HRTEM of Amorphous and Crystalline Bi Nanoparticles Prepared by Pulsed Laser Deposition
}

\author{
L.Y. Liu ${ }^{1}$, Y. T. Xing ${ }^{2}$, D. F. Franceschini ${ }^{2}$ and I. G. Solórzano ${ }^{1}$ \\ 1. DEMa, Pontifícia Universidade Católica do Rio de Janeiro, Rio de Janeiro, Brasil \\ 2. Instituto de Física, Universidade Federal Fluminense, Niterói, Brasil
}

Bulk bismuth is a very unique semimetal with a rhombohedral structure and it has special properties such as large Fermi wavelengths, strong diamagnetism, and very high magnetoresistance. It is well known that the bulk Bi does not have a superconducting transition down to $50 \mathrm{mK}$ at ambient pressure [1]. However, the Bi nanoparticles (NPs) embedded in insulator matrix [2], Bi nanowires [3] and $\mathrm{Bi} / \mathrm{Ni}$ thin films [4] show a superconducting transition. For both the Bi nanoparticles and $\mathrm{Bi} / \mathrm{Ni}$ layers, the interface plays a very important role in the superconducting transition and it needs a more detailed microstructural study at the atomic scale.

In this work Bi NPs have been prepared by pulsed laser deposition (PLD) and the microstructure has been studied by means of JEOL 2100F high resolution transmission electron microscopy (HRTEM) operating at $200 \mathrm{kV}$ under diffraction and phase contrast modes. The sample is a TEM grid with Bi NPs deposited on top. One can see from Figure 1 that the diameter of the Bi NPs is smaller than $20 \mathrm{~nm}$ and the size distribution is quite narrow. To our surprise, part of the Bi NPs is amorphous (as shown in figure 2) and part of them is crystalline (as shown in figure 3). In fact, the particles arrange themselves, as crystalline or amorphous, in different regions of the grid suggesting that surface energy component plays a significant in the aggregation mechanisms. The amorphous Bi NPs do not exhibit a clear border and the contrast (darker at the center) shows that they are spherical. In contrast, the border of the crystalline Bi NPs is clearly defined, in general with faceting morphology to maximize lower energy crystal orientations, as expected from Gibbs-Wulf construction. The lattice fringes of crystalline Bi NPs in different orientations in relation to the electron beam can be observed from the image of Fig 3 . Moreover, the distance between the atomic columns of Bi atoms in Fig. 4 is measured as $0.33 \mathrm{~nm}$ and this fits the lattice parameter of rhombohedral Bi very well.

The formation of amorphous and crystalline Bi NPs could be due to the PLD process parameters, which generates a non-homogenous plume and the expansion of it can give a different temperature in different regions, which may result in the formation of different $\mathrm{Bi}$ nanoparticles. The amorphous and crystalline $\mathrm{Bi}$ nanoparticles are expected to generate different superconducting behavior. This subject of fundamental and practical importance and is currently under investigation. [5]

\section{References:}

[1] Kurti, N.; Simon, F. E. Proc. R. Soc. London, Ser. A 1935, 151, 610.

[2] Weitzel, B.; Micklitz, H. Phys. ReV. Lett. 1991, 66, 385.

[3] Mingliang Tian et. al, Nano Letters, Vol. 6, No. 12, (November. 2006), pp. (2773-2780).

[4] LeClair, P.; Moodera, J. S.; Philip, J.; Heiman, D. Phys. Rev. Lett.2005, 94, 037006

[5] This research has been sponsored by CNPq (Brazil) and NSF -MWN(US: DMR- 0303429) under the joint Pan-American CIAM Program. The authors are grateful to PETROBRAS/ANP allowing the acquisition of the electron microscopes. 


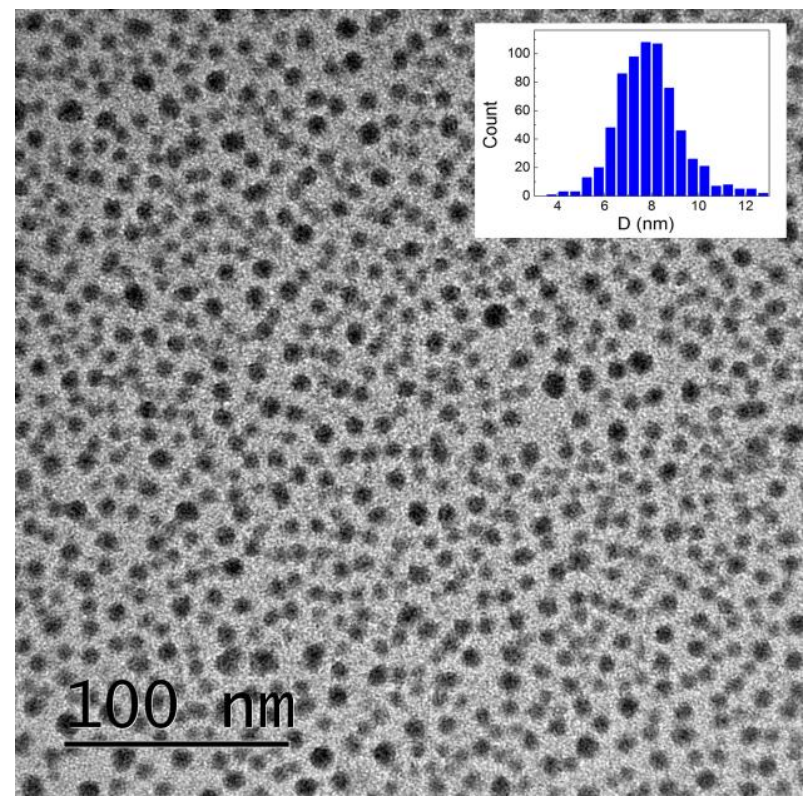

Figure 1. Low magnification image of $\mathrm{Bi}$ nanoparticles. Size distribution can be found from the inset.

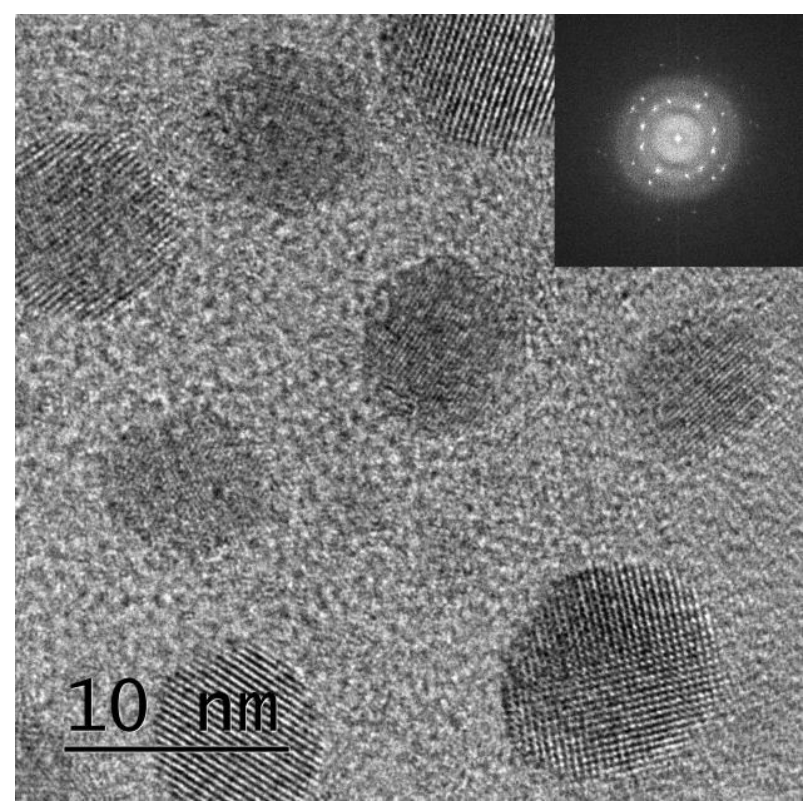

Figure 3. Crystalline Bi nanoparticles. Inset: Reduced FFT of the image.

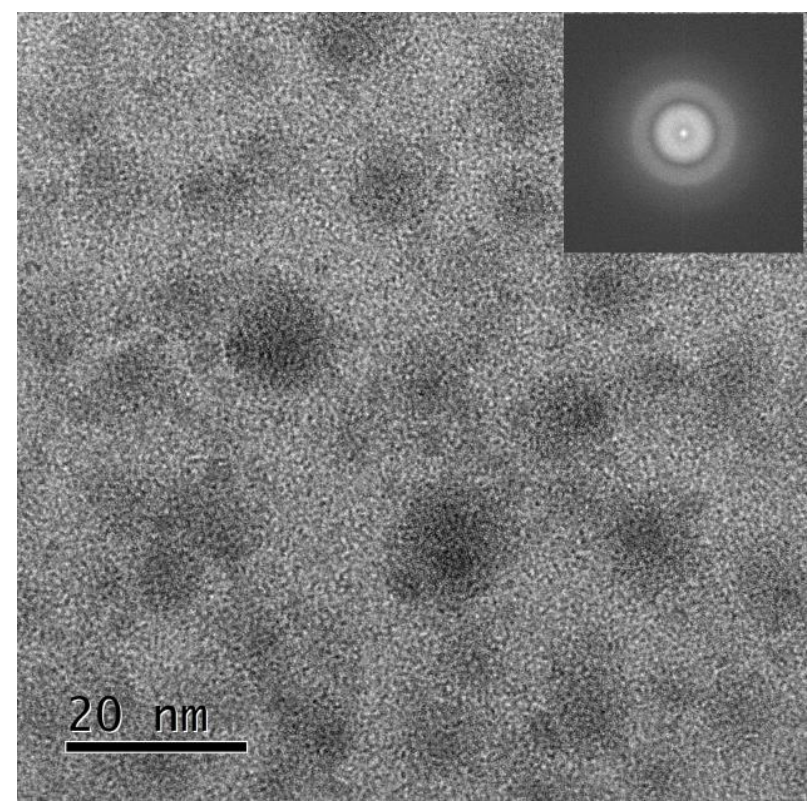

Figure 2. Amorphous Bi nanoparticles. Inset: Reduced FFT of the image.

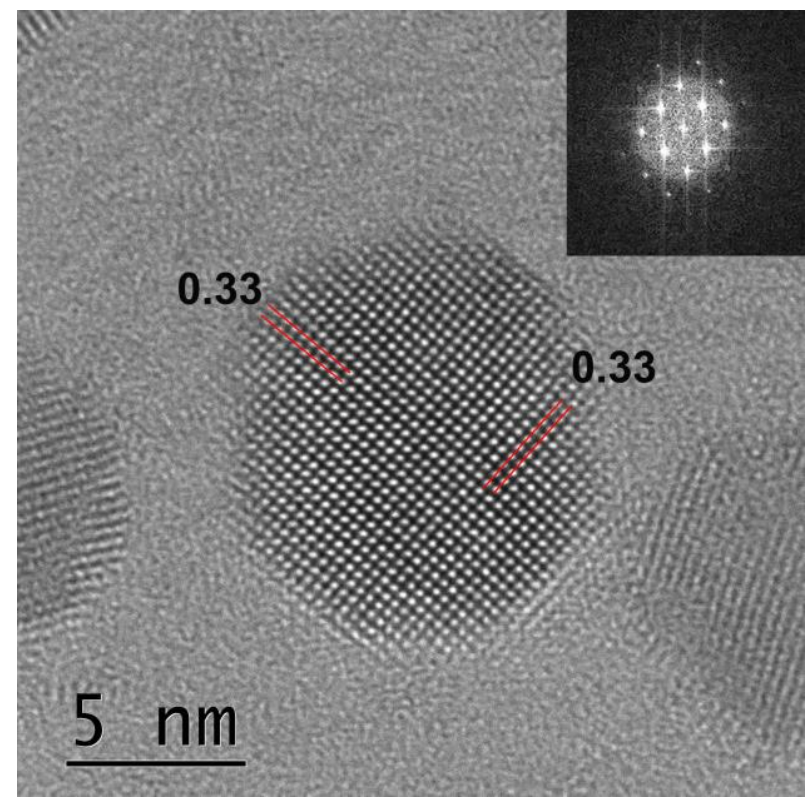

Figure 4. HRTEM image of an isolated bismuth particle. Inset: Reduced FFT of the nanoparticle 